\title{
Study of discrepancies in rubella haemagglutinin titrations and a reappraisal of diluents used in the rubella haemagglutination inhibition technique
}

\author{
HGS MURRAY, JUDITH STANTON, PS GARDNER \\ From the Central Public Health Laboratory, Division of Microbiological Reagents and Quality Control, \\ Colindale, London NW9
}

SUMMARY To elucidate inconsistencies in rubella haemagglutinin assays the components of the assay technique were examined. The results of carefully controlled assays of rubella haemagglutinin antigens from different sources in various plates and diluents with four species of indicator cells are reported. The quality and quantity of gelatin in the dextrose-gelatin-veronal buffered diluent commonly used in rubella haemagglutinin assays had a profound effect on the haemagglutination pattern and antigen titre. The veronal buffered saline used in the complement fixation test offered a valid alternative to the more complex diluents incorporating gelatin currently used in rubella haemagglutinin assays and haemagglutination inhibition tests.

Notwithstanding the recent application of passive haemadsorption, immune haemolysis, and immunosorbent techniques to rubella serology, haemagglutination inhibition (HI) remains a widely used procedure in diagnosing rubella and establishing an individual's immunity to the disease. In a limited collaborative study to define the parameters of the rubella $\mathrm{HI}$ technique with a view to establishing the procedure of choice, we were surprised to note wide discrepancies in the rubella antigen titre reported by several laboratories following a common protocol. Subsequently, a number of rubella antigens prepared and assayed in the division gave unexpectedly low titres and unstable agglutination patterns; moreover, inconsistencies were found in consecutive titrations of each of the antigens and in simultaneous titrations of an antigen in different batches of dextrose-gelatin-veronal (DGV) buffer supplemented with $0.2 \%$ bovine albumin (DGVA). These antigens gave good results in the radial haemolysis test and were of satisfactory potency in the $\mathrm{HI}$ test, though the antigen back titration did not always reflect the haemagglutinin (HA) dose used in the test.

In preliminary experiments to elucidate the anomalous HA titrations the effects of plates, diluents, and erythrocyte species on assays of freeze dried antigens from different sources were studied. The investigation was extended in two further experiments in which precautions were taken to exclude subjective bias. The observation that a particular batch of DGV buffer from a commercial source gave consistently satisfactory HA titration results led us to study the effects of the components of DGV buffer on the haemagglutination pattern. The results of all these experiments are reported below. Throughout the investigation the uniformly satisfactory HA titrations in complement fixation test diluent + $0.2 \%$ bovine albumin (CFTA) led us to recommend CFTA as an alternative to DGVA by virtue of consistent performance, ready availability, and low cost.

\section{Material and methods}

SERA

Twenty seven serum samples were tested for $\mathrm{HI}$ antibody. Six of the samples were believed to be without antibody to rubella virus; 21 were shown to have rubella antihaemagglutinin by a sensitive (overnight) rubella $\mathrm{HI}$ technique and four were from patients with rubella. Samples were stored at $-20^{\circ} \mathrm{C}$ and thawed at $37^{\circ} \mathrm{C}$ before testing.

PLATES

Polystyrene V-well Microtiter plates (Sterilin) were used. 


\section{DILUENTS}

DGVA and CFTA diluents were prepared by adding $0.2 \%$ bovine albumin fraction $\mathrm{V}$ (Armour) to DGV (commercial or prepared in DMRQC) and CFT (Oxoid Code BR16) diluents, respectively. Hepes saline albumin gelatin (HSAG) diluent (Liebhaber, 1970)' was purchased from Flow Laboratories Ltd and from Gibco-Biocult Ltd.

\section{ANTIGENS}

Antigens $23 / 80,24 / 81$, and EC83 were derived from baby hamster kidney (BHK 21) monolayer cell cultures and antigen 1/77 from BHK 21/13s suspension cultures infected with the Judith strain of rubella virus. Antigens $23 / 80,24 / 81$, and $1 / 77$ were prepared by alkaline extraction of the virus infected cells; EC83 was prepared by concentration of the haemagglutinin from the supernatants of virus infected cultures by ultrafiltration. ${ }^{2}$ All four antigens were treated with Tween 80 and ether. ${ }^{3}$ Antigens $23 / 80,24 / 81$, and $1 / 77$ were diluted in peptone water to an arbitrary HA potency and freeze dried in the presence of a stabilising agent. Antigen EC83 was stored in aliquots at $-40^{\circ} \mathrm{C}$. Antigen 79-0023, obtained from the Center for Disease Control, Atlanta, Georgia, USA, was prepared from BHK $21 / 13$ s cells infected with the Gilchrist strain of rubella virus. Antigen VR 714, derived from rubella virus strain Thomas cultivated in BHK 21 cells, was obtained from Wellcome Diagnostics.

\section{ERYTHROCYTES}

Sheep and day old chick erythrocytes preserved in Alsever's solution were purchased from Flow Laboratories Ltd. Pigeon cells were obtained from the wing vein of a pigeon and human $O$ erythrocytes were obtained from one of the authors. Both pigeon and human cells were used immediately after venepuncture. The human $O$ cells were trypsinised before use. ${ }^{4}$
HA TECHNIQUE

Duplicate $25 \mu \mathrm{l}$ serial twofold dilutions of each antigen were prepared in the specified diluent with the aid of a multichannel semi-automatic pipette (Titertek). Fifty microlitres of a $\mathbf{0 . 2 \%}$ erythrocyte suspension was added to each well with a repeater syringe (Eppendorf Multipette 4780). The cells were suspended by rotation of the plate in a Titertek Microtiter plate shaker and the plates were held at $4^{\circ} \mathrm{C}$ overnight. The titration end points were read between 30 and $60 \mathrm{~min}$ after removal of the plates from the refrigerator. The HA titre was recorded as the reciprocal of the highest dilution of antigen in which haemagglutination was complete.

\section{HI TECHNIQUE}

Rubella antihaemagglutinin was assayed by the technique recommended by the Public Health Laboratory Service Standing Advisory Committee on Viral Reagents ${ }^{5}$ with the following exceptions: HSAG and CFTA diluents were used instead of DGVA in two of the three tests carried out and the HI titre was read as the reciprocal of the highest serum dilution showing complete inhibition of haemagglutination. Day old chick erythrocytes were used in the HI tests.

\section{Results}

EFFECT OF DILUENTS, ERYTHROCYTE SPECIES, AND PLATES ON THE RUBELLA HA ASSAY

In a preliminary experiment four rubella $\mathrm{HA}$ antigens representing three manufacturers, three rubella virus strains, and two cell substrates were titrated. Dilutions from 1/16 to 1/2048 were made in Microtiter plates and erythrocytes of four species were added. The dilutions were not coded and the titrations were carried out and read by one person. The antigen titres are shown in Table 1. Duplicate titrations of one of the antigens (24/81) in a different

Table 1 Haemagglutinin titres of four rubella antigens assayed in three diluents with erythrocytes of different species

\begin{tabular}{|c|c|c|c|c|c|c|c|c|c|c|c|c|c|c|c|}
\hline \multicolumn{4}{|c|}{$\begin{array}{l}\text { Erythrocyte } 79-0023 \\
\text { species }\end{array}$} & \multicolumn{3}{|l|}{$23 / 80$} & \multicolumn{3}{|l|}{ VR 714} & \multicolumn{3}{|l|}{$24 / 81$} & \multicolumn{3}{|c|}{ Cell controls } \\
\hline & $D G V A$ & CFT & $A H S A G$ & $D G V$ & 4 CFTA & HSAG & $D G V A$ & CFTA & $H S A G$ & $D G V A$ & CFT & $A H S A G$ & $D G$ & $A$ CFTA & $H S A G$ \\
\hline $\begin{array}{l}\text { Sheep } \\
\text { Chick } \\
\text { Human } \\
\text { Pigeon }\end{array}$ & $\begin{array}{l}(8-16) \\
(64) \\
64) \\
128)\end{array}$ & $\begin{array}{l}<16 \\
128 \\
256 \\
256\end{array}$ & $\begin{array}{r}16 \\
128 \\
64 \\
512\end{array}$ & $\begin{array}{r}32 \\
128 \\
128 \\
256\end{array}$ & $\begin{array}{r}64 \\
512 \\
512 \\
1024\end{array}$ & $\begin{array}{r}16 \\
512 \\
256 \\
512\end{array}$ & $\begin{array}{l}(8-32) \\
8-256) \\
8-256) \\
(8-256)\end{array}$ & $\begin{array}{r}16 \\
512 \\
512 \\
1024\end{array}$ & $\begin{array}{r}16 \\
512 \\
128 \\
1024\end{array}$ & $\begin{array}{c}(16-64) \\
64 \\
64 \\
128\end{array}$ & $\begin{array}{r}16 \\
128 \\
128 \\
256\end{array}$ & $\begin{array}{l}\overline{32} \\
16 \\
64\end{array}$ & $\begin{array}{l}\mathrm{C} \\
\mathrm{C} \\
\mathrm{C} \\
\mathrm{C}\end{array}$ & $\begin{array}{l}\text { (C) } \\
\text { (C) } \\
\text { C) }\end{array}$ & $\begin{array}{l}\mathbf{C} \\
\mathbf{Y} \\
\mathbf{C} \\
\mathbf{Y}\end{array}$ \\
\hline
\end{tabular}

$\mathrm{C}=$ clear supernatant.

(C) = slightly discoloured supernatant.

$\mathrm{Y}=$ discoloured supernatant.

Brackets indicate collapsed haemagglutinin patterns.

DGVA = dextrose-gelatin-veronal buffer supplemented with $0.2 \%$ bovine albumin.

CFTA $=$ complement fixation test diluent supplemented with $0.2 \%$ bovine albumin.

HSAG $=$ hepes saline albumin gelatin diluent. 
Table 2 Haemagglutinin titres of two rubella antigens assayed in various diluents

\begin{tabular}{|c|c|c|c|c|}
\hline \multirow{2}{*}{ Diluent } & \multicolumn{2}{|l|}{$V R 714$} & \multicolumn{2}{|l|}{$I / 77 F$} \\
\hline & Chick & Pigeon & Chick & Pigeon \\
\hline $\begin{array}{l}\text { DGVA (commercial A) } \\
\text { DGVA (commercial B) } \\
\text { DGVA DMRQC } \\
\text { CFTA Oxoid } \\
\text { HSAG Gibco } \\
\text { HSAG Flow }\end{array}$ & $\begin{array}{l}(<8-32) \\
\mathrm{L} \\
(8) \\
512 \\
512 \\
512\end{array}$ & $\begin{array}{l}(8) \\
\mathrm{L} \\
(8) \\
>1024 \\
(128) \\
>1024\end{array}$ & $\begin{array}{l}(8-16) \\
\mathrm{L} \\
(16) \\
128 \\
128 \\
128\end{array}$ & $\begin{array}{l}(8) \\
L \\
(16) \\
256 \\
128 \\
128\end{array}$ \\
\hline
\end{tabular}

Brackets indicate collapsed haemagglutination patterns. $\mathrm{L}=$ lysis.

For other abbreviations see Table 1.

batch of plates from those used in the main test gave identical results. Collapsed haemagglutination patterns in DGVA diluent rendered the titrations of 79-0023 and VR 714 antigens virtually unreadable. With the exception of $24 / 81$ the antigen titrations in HSAG diluents were satisfactory with pigeon, chick, and human cells, while the highest and most consistent antigen titres, irrespective of erythrocyte species, were found in CFTA diluent. In general, pigeon cells were more susceptible to haemagglutination than day old chick or trypsinised human $O$ erythrocytes. Sheep cells were relatively insensitive. Cell controls were satisfactory except for discoloured supernatants in the chick and pigeon cell controls in HSAG diluent.

The influence of the diluent in HA titrations was further studied in an experiment designed to minimise subjective bias influencing the results. Two antigens, one commercial and the other from DMRQC, were titrated with both day old chick and pigeon erythrocytes as indicator cells. Duplicate tests were carried out by two of us working independently from a common protocol. Diluents were assigned an arbitrary designation by a colleague, who retained the code until the results of the titrations were recorded. Comparable results were obtained by both workers and end points read by each of the authors were usually identical and never differed by more than one dilution step; the consensus of the three readings was therefore taken as the titre (Table 2). Microscopical examination of the contents of the wells containing one of the DGVA diluents showed that the erythrocytes had lysed. Collapsed haemagglutination patterns and a gradual transition from partial agglutination rendered the titrations in the isotonic DGVA diluents difficult or impossible to read. Unequivocal end points were invariably obtained in CFTA diluent.

In a supplementary experiment, two Tween $^{80}$ ether treated rubella antigens derived from BHK 21 cell cultures infected with rubella virus (strain Judith) were titrated in CFTA and HSAG diluents with day old chick erythrocytes as indicator cells. Antigen 24/81 was obtained by alkaline extraction of the virus infected cells and antigen EC83 was obtained by concentration of the supernatants from the cultures. In the case of the extracellular antigen the same HA titre was obtained in both diluents whereas the cell associated antigen showed a significantly lower titre in HSAG diluent (Table 3 ).

The main objective of the fourth experiment was to determine the influence of the plate surface on the HA titre of the antigen and on the quality of the haemagglutination pattern. Three rubella antigens were titrated in new plates, in new plates given a preliminary rinse in deionised water, and in plates which had been reused several times. It was thought that the wells of the recycled plates, roughened by the use of Takatsy diluters, ${ }^{6}$ might provide a more favourable surface for the formation of a haemagglutination pattern than the smooth surface of new plates. Duplicate serial twofold dilutions of each antigen were prepared with a multichannel pipette in three coded diluents in one half of each plate. Identical dilutions of the antigens were made with Takatsy diluters in the remaining half of the plate. Day old chick erythrocytes were added and the test read after overnight settling at $4^{\circ} \mathrm{C}$. As in the previous experiment the complete test was done in duplicate by two of us working independently from a common protocol and all the titrations were read by each of the authors. After decoding, antigen titres in CFTA and HSAG diluents were found to be virtually identical and the titration end points recorded by the readers were in close accord. The results of assays performed with Takatsy diluters were not significantly different from those carried out with the multichannel pipette; consensus reading of the four antigen titrations in a particular plate and diluent was therefore recorded as the titre (Table 4). Antigen titres in DGVA showed considerable variation and were often difficult to read as shown by the range of values embracing the probable titre recorded in Table 4. Except for VR 714, in which titres in HSAG and CFTA diluents were comparable, the highest antigen titres were found in CFTA diluent irrespective of plate history. Contrary to expectation titration end points tended to be lower in used plates than in new plates.

Table 3 Haemagglutinin titres of two rubella antigens assayed in two diluents

\begin{tabular}{lll}
\hline Diluent & $24 / 81$ & EC83 \\
\hline CFTA & 128 & 256 \\
HSAG & 32 & 256 \\
\hline
\end{tabular}

For abbreviations see Table 1 . 
Study of discrepancies in rubella haemagglutinin titrations

Table 4 Haemagglutinin titres of three rubella antigens assayed in various plates and diluents

\begin{tabular}{|c|c|c|c|c|c|c|c|c|c|}
\hline \multirow[t]{2}{*}{ Plate history } & \multicolumn{3}{|l|}{$V R 714$} & \multicolumn{3}{|l|}{$24 / 81$} & \multicolumn{3}{|l|}{$1 / 77 F$} \\
\hline & $D G V A$ & CFTA & $H S A G$ & $D G V A$ & CFTA & $H S A G$ & $D G V A$ & CFTA & $H S A G$ \\
\hline $\begin{array}{l}\text { New (untreated) } \\
\text { New (rinsed) } \\
\text { Used }\end{array}$ & $\begin{array}{l}(32-128) \\
(32-64) \\
(32-128)\end{array}$ & $\begin{array}{l}256 \\
256 \\
256\end{array}$ & $\begin{array}{r}128 \\
128 \\
64\end{array}$ & $\begin{array}{l}(32-128) \\
(32) \\
(64)\end{array}$ & $\begin{array}{l}128 \\
64-128 \\
64\end{array}$ & $\begin{array}{r}16 \\
16 \\
<16\end{array}$ & $\begin{array}{l}(32-128) \\
(32) \\
(32)\end{array}$ & $\begin{array}{r}128 \\
64 \\
64\end{array}$ & $\begin{array}{l}32 \\
32 \\
16\end{array}$ \\
\hline
\end{tabular}

Brackets indicate collapsed haemagglutinin patterns.

For abbreviations see Table 1.

Fortuitously, at the end of the work already reported, satisfactory rubella HA titrations were obtained in a batch of DGV buffer, supplemented with $0.2 \%$ bovine albumin, from one of the manufacturers whose product had previously given the familiar unstable granular haemagglutination pattern. Consequently, we attempted to determine whether the mode of preparation of DGV buffer or qualitative or quantitative variations in one or more of its components could affect the haemagglutination pattern. After preliminary experiments to establish that the phenomenon was not related to filtration of the diluent through a $0.22 \mu \mathrm{m}$ membrane or variations in the concentration of hydrogen ions or divalent cations, the qualitative and quantitative effects of gelatin were examined. Gelatin was selected for scrutiny since it is an undefined substance and the other components of DGV were available as analytical reagents of specified purity. Accordingly, DGV buffers were prepared with gelatins from two manufacturers. One gelatin dissolved easily in water at $37^{\circ} \mathrm{C}$ to provide a solution that filtered rapidly through a $0.22 \mu \mathrm{m}$ membrane. The other gelatin dissolved with difficulty and the resulting solution filtered relatively slowly. HA antigen titrations in the DGVA diluents incorporating each of the gelatins gave the characteristic granular collapsing agglutination pattern and a twofold difference in HA titre. It was found, with either buffer, that the unstable haemagglutination pattern was directly related to the concentration of gelatin in the diluent. When gelatin was omitted from the dextrose-veronal buffer, the haemagglutination pattern in the diluent formed by the addition of $0.2 \%$ albumin (DVA) was satisfactory and virtually indistinguishable from the agglutination pattern in CFTA. Cell controls and the wells beyond the titration end points in both DVA and CFTA diluents showed distinct buttons of unagglutinated cells but the supernatants were discoloured because some of the erythrocytes remained in suspension. The addition of as little as $10 \mathrm{mg} / \mathrm{l}$ of gelatin to either diluent resulted in clear supernatants and well defined cell buttons at the expense of a slight fall in HA titre. Increasing the concentration of gelatin gave rise to a dose dependent degradation of the agglutination pattern and a progressive reduction in HA titre.

EVALUATION OF CFTA DILUENT IN THE HI TEST

The sera specified under the Material and Methods section were titrated by the $\mathrm{HI}$ test in DGVA, CFTA, and HSAG diluents. Six sera had titres $<10$ in all the diluents. Titres in all three diluents were in close agreement. Most titrations in DGVA diluent were clear and the end points were easily read. In a few tests, however, the end points were equivocal because of faint partial agglutination. The supernatants in the titrations, with CFTA diluent were slightly discoloured but the end points were unequivocal and easily read. Moreover, titration end points in CFTA were not bedevilled by incomplete agglutination as they were in DGVA diluent.

\section{Discussion}

Numerous diluents have been recommended for use in the rubella HI test. The adverse effect of excess gelatin on rubella haemagglutination ${ }^{178}$ was amply confirmed in the present study. Furthermore, variation in the quality of gelatins from different sources, analogous to variation in albumins, ${ }^{8}$ influenced the HA titre. Although the concentration of gelatin in HSAG diluent is low, 'the discrepant assay results in the two HSAG diluents may reflect different gelatins or albumins used in their preparation. The discrepant titres given by one of the alkaline extracted rubella antigens in HSAG and CFTA diluents exemplifies the unexplained interdependence of antigen and diluent noted by Schmidt and Lennette ${ }^{7}$ in a comprehensive evaluation of the factors affecting rubella $\mathrm{HA}$ titrations.

Notwithstanding the failure of unagglutinated chick erythrocytes to settle completely in gelatin free diluent, titration end points in CFTA diluent were unequivocal and reproducible, the haemagglutination pattern was stable and fine grained, and unagglutinated chick erythrocytes settled rapidly to form well defined buttons.

HA titres were usually higher in CFTA diluent than in HSAG and equally independent of erythro- 
cyte species. Furthermore, optimal HA titres were obtained in CFTA diluent irrespective of the rubella virus strain used, the BHK cell substrate in which the virus was cultivated, or the intra or extracellular origin of the virus from which the haemagglutinin was prepared. CFT diluent is chemically defined and available in a convenient tablet form. In our experience DGVA is an unsuitable diluent for rubella HA and $\mathrm{HI}$ assays because of its high gelatin content. We recommend the use of CFT diluent, supplemented with an albumin that has no inhibitory effect on rubella haemagglutinin titre, as the diluent of choice for rubella $\mathrm{HA}$ and $\mathrm{HI}$ tests.

\section{References}

' Liebhaber H. Measurement of rubella antibody by hemagglutination inhibition. J Immunol 1970; 104:818-25

${ }^{2}$ Trudel M, Payment P. Concentration and purification of rubella virus hemagglutinin by hollow fiber ultrafiltration and sucrose density centrifugation. Can J Microbiol 1980;26:1334-9.

${ }^{3}$ Norrby E. Haemagglutination by measles virus. 4. A simple pro- cedure for production of high potency antigen for haemagglutination inhibition (HI) tests. Proc Soc Exp Biol Med 1962;111:814-8.

4 CDC Manual. Serodiagnosis of: toxoplasmosis, rubella, cytomegalic inclusion disease, herpes simplex. Immunology Series no 5, Procedural Guide. Washington DC: US Department of Health, Education, and Welfare, 1974.

${ }^{s}$ Report. Haemagglutination-inhibition test for the detection of rubella antibody. J Hyg 1978;81:373-82.

- Takatsy G. The use of spiral loops in serological and virological micro-methods. Acta Microbiol 1956;3:1.91.

' Schmidt NJ, Lennette EH. Variables of the rubella hemagglutination-inhibition test system and their effect on antigen and antibody titres. Am Soc Microbiol 1970;19:491504.

- Campbell JB, Grunberger T, Ellins ML. Influence of albumin on rubella hemagglutination and the hemagglutination-inhibition test. Can J Microbiol 1975;21:1172-7.

Requests for reprints to: Judith Stanton, Division of Microbiological Reagents and Quality Control, Central Public Health Laboratory, Colindale Avenue, London NW9 5HT, England. 